\title{
$\ln 2016$
}

How it felt to live in the Arab World five vears after the "Arab Sprina»

\section{Avatar document}

(needed as temporary DOI placeholder)

This avatar will soon be replaced with the actual In 2016 entry. The latter is published already in the full text version of the entire In 2016 collection, containing all individual entries: 